\title{
Same-sign tetralepton signature at the Large Hadron Collider and a future $p p$ collider
}

\author{
Eung Jin Chun, ${ }^{1, *}$ Sarif Khan, ${ }^{2,3, \dagger}$ Sanjoy Mandal ${ }^{4,5, \ddagger}$ Manimala Mitra, ${ }^{4,5, \S}$ and Sujay Shil $\oplus^{4,5, \uparrow}$ \\ ${ }^{1}$ Korea Institute for Advanced Study, Seoul 130-722, Korea \\ ${ }^{2}$ Institut für Theoretische Physik, Georg-August-Universität Göttingen, \\ Friedrich-Hund-Platz 1, Göttingen, D-37077 Germany \\ ${ }^{3}$ Harish-Chandra Research Institute, Allahabad, India \\ ${ }^{4}$ Institute of Physics, Sachivalaya Marg, Bhubaneswar, Odisha 751005, India \\ ${ }^{5}$ Homi Bhabha National Institute, BARC Training School Complex, \\ Anushakti Nagar, Mumbai 400094, India
}

(Received 9 December 2019; accepted 10 February 2020; published 7 April 2020)

\begin{abstract}
We analyze a novel signature of the type-II seesaw model-a same-sign tetralepton signal arising from the mixing of neutral Higgs bosons and their subsequent decays to singly and doubly charged Higgs bosons. For this, we consider wide ranges of the triplet vacuum expectation value (vev) and Yukawa couplings that are consistent with the observed neutrino masses and mixing as well as the LHC search limits. We find that a doubly charged Higgs boson with mass around $250 \mathrm{GeV}$ and triplet vev around $10^{-4}-10^{-2} \mathrm{GeV}$ can give a significantly large number of events through its decay to same-sign $W$ gauge bosons at the high-luminosity LHC with $3000 \mathrm{fb}^{-1}$ of data. We also pursue the analysis for a future hadron collider with the c.m. energy of $100 \mathrm{TeV}$. Considering a heavy Higgs boson of around $900 \mathrm{GeV}$ and an intermediate region of the triplet vev, where both same-sign dilepton and gauge boson decays can occur, we identify a limited range of the parameters where the number of same-sign tetralepton events are as large as 1000.
\end{abstract}

DOI: 10.1103/PhysRevD.101.075008

\section{INTRODUCTION}

After the discovery of the Standard Model (SM) Higgs boson, one of the key questions that still remains unexplained is the origin of light neutrino masses and mixings. A number of neutrino oscillation experiments have observed that the solar and atmospheric neutrino mass splittings are $\Delta m_{12}^{2} \sim 10^{-5} \mathrm{eV}^{2}$ and $\Delta m_{13}^{2} \sim 10^{-3} \mathrm{eV}^{2}$. The PontecorvoMaki-Nakagawa-Sakata (PMNS) mixing angles are $\theta_{12} \sim 32^{\circ}, \theta_{23} \sim 45^{\circ}$, and $\theta_{13} \sim 9^{\circ}$ [1]. Once we include the right-handed neutrinos in the theory, a Dirac mass term can be generated for light neutrinos. However, to generate $\mathrm{eV}$ neutrino masses, this requires a very large hierarchy of the Yukawa couplings $Y_{\nu} \sim \mathcal{O}\left(10^{-11}\right)$ within the SM. The light neutrinos being electromagnetic charge neutral can be a Majorana particle, and their masses can have a different

\footnotetext{
*ejchun@kias.re.kr

sarif.khan@uni-goettingen.de

smandal@iopb.res.in

\$manimala@iopb.res.in

"sujayshil1@gmail.com
}

Published by the American Physical Society under the terms of the Creative Commons Attribution 4.0 International license. Further distribution of this work must maintain attribution to the author(s) and the published article's title, journal citation, and DOI. Funded by SCOAP. origin compared to the other SM fermions. One of the profound mechanisms to generate Majorana masses of light neutrinos is the seesaw, where tiny $\mathrm{eV}$ masses of the Majorana neutrinos are generated from the lepton number violating $d=5$ operator $L L H H / \Lambda[2,3]$. There can be different UV completed theories behind this operator, commonly known as type-I, -II, and -III seesaw mechanisms. These different models accommodate extensions of the $\mathrm{SM}$ fermion/scalar contents by $S U(2)_{L}$ singlet fermions [4-10], $S U(2)_{L}$ triplet scalar boson [11-14], and $S U(2)_{L}$ triplet fermions [15], respectively. Among these, the type-II seesaw model, where a triplet scalar field with the hypercharge $Y=+2$ is added to the SM, has an extended scalar sector. See [16-18] for the details of the Higgs spectrum. The bound from a vacuum stability, perturbativity, and electroweak precision test has been studied in [19]. The neutral component of the triplet acquires a vacuum expectation value (vev) $v_{\Delta}$ and generates $\mathrm{eV}$ scale neutrino masses through the Yukawa interaction between the lepton doublets and triplet Higgs field. The same Yukawa interaction also has a large impact on the charged Higgs phenomenology in this model. The presence of a doubly charged Higgs that can have distinct decay modes whose branching ratios are determined by the observed neutrino oscillation data [20] is the most appealing feature of this model. Hence, a 
discovery of this exotic particle will be a smoking gun signature of this model.

A number of searches have been carried out to look for the signature of the doubly charged Higgs at collider and noncollider experiments [20]. See [21] for the Tevatron and [22-34] for the LHC [35-37] for HE-LHC and future hadron colliders. Depending on the triplet vev, the doubly charged Higgs boson can decay via distinguished decays modes. Assuming degenerate charged Higgs masses, it decays predominantly to same-sign dileptons (gauge bosons) for $v_{\Delta}<(>) 10^{-4} \mathrm{GeV}$. For nondegenerate charged Higgs, in the intermediate range of triplet vev, the cascade decay to singly charged Higgs can also be dominant and has been explored in [22-24]. The CMS and ATLAS Collaborations have searched for the same-sign dilepton final states and constrained the mass of the doubly charged Higgs as $M_{H^{ \pm \pm}}>820,870 \mathrm{GeV}$ at $95 \%$ C.L. $[38,39]$ assuming $\operatorname{Br}\left(H^{ \pm \pm} \rightarrow \ell^{ \pm} \ell^{ \pm}\right)=100 \%$. Additionally, ATLAS also searched pair production of doubly charged Higgs with subsequent decays into gauge bosons [40]. This search constrained the mass of doubly charged Higgs $M_{H^{ \pm \pm}}$ between 200 and $220 \mathrm{GeV}$ at 95\% C.L., relevant for $v_{\Delta}>10^{-4} \mathrm{GeV}$. The vector boson fusion channel, where the $H^{ \pm \pm}$is produced in association with two jets, gives relaxed constraints $[41,42]$. The collider signatures and the discovery prospect of this scenario have been discussed in [43-49]. Previous searches for $H^{ \pm \pm}$in the pair-production channel and their subsequent decays into same-sign leptons at LEP-II has put a constraint $M_{H^{ \pm \pm}}>97.3 \mathrm{GeV}$ at 95\% C.L. [50]. For the earlier discussions on the Higgs triplet model at a linear collider, see [51-55].

Most of the works in the literature explored the dilepton or gauge boson decay modes of the doubly charged Higgs leading to multilepton final states. Because of the possible cascade decays of the charge neutral Higgs into a singly charged Higgs and the cascade decay of a singly charged Higgs into a doubly charged Higgs, the model can also lead to a very unique signature, same-sign tetralepton final states. This was first proposed in [56] and explored for the lower triplet vev, where dilepton decay is predominant. In this work, we consider a wide range of the triplet vev, particularly focusing on gauge boson decay modes, and explore the signature for $14 \mathrm{TeV}$ LHC. For the higher range of the triplet vev, as the LHC constraint on the mass of doubly charged Higgs is relatively relaxed [40], we therefore perform the analysis for the lighter Higgs state, as low as $M_{H^{ \pm \pm}} \sim 247 \mathrm{GeV}$. In addition, we also consider a very high energy $p p$ collider that can operate with c.m. energy $\sqrt{s}=100 \mathrm{TeV}$ and explore this unique signature for a heavy doubly charged Higgs. We show that for heavier doubly charged Higgs, there is a very narrow region of the triplet vev, which can accommodate significantly large $\mathcal{O}\left(10^{3}\right)$ same-sign tetralepton signatures.

Our paper is organized as follows: We briefly review the basics of the type-II seesaw model in Sec. II. In Sec. III, we discuss the branching ratios of doubly and singly charged Higgs and the relation between $H^{ \pm \pm}$and $H^{ \pm}$decays. In Secs. IV and V, we present the simulation of samesign tetralepton signal at $\sqrt{s}=14 \mathrm{TeV}$ LHC and $\sqrt{s}=$ $100 \mathrm{TeV}$. Finally, we present our conclusions in Sec. VI.

\section{MODEL DESCRIPTION}

One of the most simplest seesaw models is the type-II seesaw model [11-14], which, in addition to the SM particle contents, also contains one $S U(2)_{L}$ triplet Higgs field

$$
\Delta=\left(\begin{array}{cc}
\frac{\Delta^{+}}{\sqrt{2}} & \Delta^{++} \\
\Delta^{0} & -\frac{\Delta^{+}}{\sqrt{2}}
\end{array}\right) \sim(1,3,2) .
$$

The neutral components of the SM doublet $(\Phi)$ and triplet Higgs fields are denoted as $\Phi^{0}=\frac{1}{\sqrt{2}}\left(\phi^{0}+i \chi^{0}\right)$ and $\Delta^{0}=\frac{1}{\sqrt{2}}\left(\delta^{0}+i \eta^{0}\right)$, respectively. The neutral component of $\Delta$ acquires vev and generates Majorana masses for light neutrinos. We denote the vev's of $\phi^{0}$ and $\delta^{0}$ by $v_{\Phi}$ and $v_{\Delta}$, where $v^{2}=v_{\Phi}^{2}+v_{\Delta}^{2}=(246 \mathrm{GeV})^{2}$. The kinetic term for the triplet has the following form

$$
\mathcal{L}_{\text {kin }}(\Delta)=\operatorname{Tr}\left[\left(D_{\mu} \Delta\right)^{\dagger}\left(D^{\mu} \Delta\right)\right] .
$$

In the above, $D_{\mu}$ is the covariant derivative $D_{\mu} \Delta=$ $\partial_{\mu} \Delta+i \frac{g}{2}\left[\tau^{a} W_{\mu}^{a}, \Delta\right]+i g^{\prime} B_{\mu} \Delta$. The new triplet scalar field $\Delta$ being a triplet under $S U(2)_{L}$ interacts with the SM gauge bosons. In addition to the kinetic term, $\Delta$ has Yukawa interaction with the SM lepton doublet. The Yukawa interactions of $\Delta$ with the lepton fields are

$$
\mathcal{L}_{Y}(\Phi, \Delta)=Y_{\Delta} \overline{L_{L}^{c}} i \tau_{2} \Delta L_{L}+\text { H.c. },
$$

where $Y_{\Delta}$ is a $3 \times 3$ matrix and $c$ denotes charge conjugation. The scalar potential of the Higgs fields $\Phi$ and $\Delta$ is

$$
\begin{aligned}
V(\Phi, \Delta)= & m_{\Phi}^{2} \Phi^{\dagger} \Phi+\tilde{M}_{\Delta}^{2} \operatorname{Tr}\left(\Delta^{\dagger} \Delta\right)+\left(\mu \Phi^{T} i \tau_{2} \Delta^{\dagger} \Phi+\text { H.c. }\right) \\
& +\frac{\lambda}{4}\left(\Phi^{\dagger} \Phi\right)^{2}+\lambda_{1}\left(\Phi^{\dagger} \Phi\right) \operatorname{Tr}\left(\Delta^{\dagger} \Delta\right)+\lambda_{2}\left[\operatorname{Tr}\left(\Delta^{\dagger} \Delta\right)\right]^{2} \\
& +\lambda_{3} \operatorname{Tr}\left[\left(\Delta^{\dagger} \Delta\right)^{2}\right]+\lambda_{4} \Phi^{\dagger} \Delta \Delta^{\dagger} \Phi,
\end{aligned}
$$

where $m_{\Phi}$ and $\tilde{M}_{\Delta}$ are real parameters with mass dimension one, and $\lambda, \lambda_{1-4}$ are dimensionless quartic Higgs couplings. Note that $\mu$ is the parameter with positive mass dimension. The triplet field $\Delta$ carries lepton number +2 , and hence, the Yukawa term conserves lepton number. However, the lepton number is violated two units by a nonzero $\mu$. Therefore, together a nonzero $\mu$ and a nonzero $Y_{\nu}$ violate lepton number symmetry.

The scalar potential that generates the scalar mass matrix includes trilinear as well as quartic couplings among the 
scalar fields. The scalar mass matrix, after diagonalization, generates seven physical Higgs states. They are the charged Higgs bosons $H^{ \pm \pm}, H^{ \pm}$, and the neutral Higgs bosons $h^{0}$, $H^{0}$, and $A^{0}$. The two charged scalar fields $\Phi^{ \pm}$of $\Phi$ and $\Delta^{ \pm}$ of $\Delta$ mix to give singly charged states $H^{ \pm}$and the charged Goldstone $\chi^{ \pm}$bosons. Similarly, the mixing between the two charge-parity- $(C P)$ odd fields $\left(\chi^{0}\right.$ and $\left.\eta^{0}\right)$ gives rise to $A^{0}$ and the neutral Goldstone boson $\rho^{0}$. Finally, we obtain the SM Higgs boson $(h)$ and a heavy Higgs boson $(H)$ via the mixing of the two neutral $C P$-even states $\Phi^{0}$ and $\delta^{0}$. For the detail description of the charged and neutral mass matrix, see [16].

The minimization conditions of the potential are

$$
\frac{\partial V(\Phi, \Delta)}{\partial v_{\Phi}}=0, \quad \frac{\partial V(\Phi, \Delta)}{\partial v_{\Delta}}=0 .
$$

These give the following conditions for $m_{\Phi}^{2}, M^{2}$ :

$$
\begin{gathered}
m_{\Phi}^{2}=\frac{1}{2}\left[-\frac{v_{\Phi}^{2} \lambda}{2}-v_{\Delta}^{2}\left(\lambda_{1}+\lambda_{4}\right)+2 \sqrt{2} \mu v_{\Delta}\right], \\
\tilde{M}^{2}=M_{\Delta}^{2}-\frac{1}{2}\left[2 v_{\Delta}^{2}\left(\lambda_{2}+\lambda_{3}\right)+v_{\Phi}^{2}\left(\lambda_{1}+\lambda_{4}\right)\right], \\
\text { with } \quad M_{\Delta}^{2} \equiv \frac{v_{\Phi}^{2} \mu}{\sqrt{2} v_{\Delta}}
\end{gathered}
$$

The diagonalization conditions for the neutral and charged scalar fields are

$$
\begin{aligned}
\left(\begin{array}{l}
\phi^{ \pm} \\
\Delta^{ \pm}
\end{array}\right) & =\left(\begin{array}{cc}
\cos \beta_{ \pm} & -\sin \beta_{ \pm} \\
\sin \beta_{ \pm} & \cos \beta_{ \pm}
\end{array}\right)\left(\begin{array}{l}
\chi^{ \pm} \\
H^{ \pm}
\end{array}\right), \\
\left(\begin{array}{l}
\chi \\
\eta
\end{array}\right) & =\left(\begin{array}{cc}
\cos \beta_{0} & -\sin \beta_{0} \\
\sin \beta_{0} & \cos \beta_{0}
\end{array}\right)\left(\begin{array}{l}
\rho^{0} \\
A^{0}
\end{array}\right), \\
\left(\begin{array}{c}
\phi^{0} \\
\delta^{0}
\end{array}\right) & =\left(\begin{array}{cc}
\cos \alpha & -\sin \alpha \\
\sin \alpha & \cos \alpha
\end{array}\right)\left(\begin{array}{c}
h^{0} \\
H^{0}
\end{array}\right),
\end{aligned}
$$

where the mixing angles

$$
\begin{aligned}
& \tan \beta_{ \pm}=\frac{\sqrt{2} v_{\Delta}}{v_{\Phi}}, \quad \tan \beta_{0}=\frac{2 v_{\Delta}}{v_{\Phi}}, \\
& \tan 2 \alpha=\frac{4 v_{\Delta}}{v_{\Phi}} \frac{v_{\Phi}^{2}\left(\lambda_{1}+\lambda_{4}\right)-2 M_{\Delta}^{2}}{v_{\Phi}^{2} \lambda-2 M_{\Delta}^{2}-4 v_{\Delta}^{2}\left(\lambda_{2}+\lambda_{3}\right)} .
\end{aligned}
$$

All these mixings being proportional to the ratio of $\frac{v_{\Delta}}{v_{\Phi}}$ are very small.

The physical masses of the doubly and singly charged Higgs bosons $H^{ \pm \pm}$and $H^{ \pm}$can be written as

$$
\begin{aligned}
& m_{H^{++}}^{2}=M_{\Delta}^{2}-v_{\Delta}^{2} \lambda_{3}-\frac{\lambda_{4}}{2} v_{\Phi}^{2}, \\
& m_{H^{+}}^{2}=\left(M_{\Delta}^{2}-\frac{\lambda_{4}}{4} v_{\Phi}^{2}\right)\left(1+\frac{2 v_{\Delta}^{2}}{v_{\Phi}^{2}}\right) .
\end{aligned}
$$

The $C P$-even and $C P$-odd neutral Higgs bosons $h$ and $H$ have the physical masses

$$
\begin{aligned}
& m_{h^{0}}^{2}=\mathcal{T}_{11}^{2} \cos ^{2} \alpha+\mathcal{T}_{22}^{2} \sin ^{2} \alpha-\mathcal{T}_{12}^{2} \sin 2 \alpha, \\
& m_{H^{0}}^{2}=\mathcal{T}_{11}^{2} \sin ^{2} \alpha+\mathcal{T}_{22}^{2} \cos ^{2} \alpha+\mathcal{T}_{12}^{2} \sin 2 \alpha .
\end{aligned}
$$

In the above, $\mathcal{T}_{11}, \mathcal{T}_{22}$, and $\mathcal{T}_{12}$ have the following expressions:

$\mathcal{T}_{11}^{2}=\frac{v_{\Phi}^{2} \lambda}{2}, \quad \mathcal{T}_{22}^{2}=M_{\Delta}^{2}+2 v_{\Delta}^{2}\left(\lambda_{2}+\lambda_{3}\right)$,

$\mathcal{T}_{12}^{2}=-\frac{2 v_{\Delta}}{v_{\Phi}} M_{\Delta}^{2}+v_{\Phi} v_{\Delta}\left(\lambda_{1}+\lambda_{4}\right)$.

The $C P$-odd Higgs field $A^{0}$ has the following mass

$$
m_{A}^{2}=M_{\Delta}^{2}\left(1+\frac{4 v_{\Delta}^{2}}{v_{\Phi}^{2}}\right), \quad \text { with } \quad M_{\Delta}^{2}=\frac{v_{\Phi}^{2} \mu}{\sqrt{2} v_{\Delta}} .
$$

The difference between the $H^{ \pm \pm}$and $H^{ \pm}$masses is dictated by the coupling $\lambda_{4}$ of the scalar potential. For a positive $\lambda_{4}$, the $H^{ \pm \pm}$is lighter than $H^{ \pm}$. The mass difference $\Delta M^{2}$ is

$$
\Delta M^{2}=M_{H^{ \pm}}^{2}-M_{H^{ \pm \pm}}^{2} \sim \frac{\lambda_{4}}{2} v_{\Phi}^{2}+\mathcal{O}\left(v_{\Delta}^{2}\right) .
$$

Throughout our analysis, we consider the mass hierarchy $M_{H^{ \pm \pm}}<M_{H^{ \pm}}$. Among the neutral Higgs fields, we identify $h^{0}$ as the SM Higgs with mass $M_{h^{0}}=125 \mathrm{GeV}$. The mass of $h^{0}$ is primarily decided by $\lambda$, where the mass of $H^{0}$ is primarily decided by $M_{\Delta}$. The neutral Higgs mixing angle $\alpha$ is very small, and hence, $\cos \alpha \simeq 1$. On the other hand, the charged Higgs and $C P$-odd Higgs mixing angles $\tan \beta_{ \pm}$ and $\tan \beta_{0}$ being proportional $v_{\Delta} / v_{\Phi}$ are very small, $\tan \beta \sim 10^{-3}$. Note that the mass squared difference between $H^{ \pm}$and $H^{0}$ in the limit $v_{\Delta}<v_{\Phi}$ is

$$
M_{H^{0}}^{2}-M_{H^{ \pm}}^{2} \sim \lambda_{4} \frac{v_{\Phi}^{2}}{4}+\mathcal{O}\left(v_{\Delta}^{2}\right) .
$$

Therefore, the mass difference between $M_{H^{ \pm \pm}}, M_{H^{ \pm}}$and the mass difference between $M_{H^{0}}, M_{H^{ \pm}}$are almost similar and dictated by the same set of parameters $\lambda_{4}$ and electroweak vev $v_{\Phi}$. The mass squared difference between $H^{0}$ and $A^{0}$ is extremely small, as this is proportional to the triplet vev, 


$$
M_{H^{0}}^{2}-M_{A^{0}}^{2} \sim 2 v_{\Delta}^{2}\left(\lambda_{2}+\lambda_{3}\right)-\frac{4}{\sqrt{2}} \mu v_{\Delta} .
$$

We denote the mass difference between $H^{0}$ and $A^{0}$ by $M_{H^{0}}-M_{A^{0}} \sim \delta M \sim v_{\Delta}$, and the mass difference between $H^{ \pm}$and $H^{0}$ by $M_{H^{ \pm}}-M_{H^{0}} \sim \Delta M$. As we will discuss in the next subsequent sections, the latter parameter is important for a few of the decay modes that depend on charged Higgs and neutral Higgs mass splitting and is one of the key parameters for our discussion.

Because of the nontrivial representations of $\Delta$, the Higgs triplet has interactions with a number of SM fermions and gauge bosons. This opens up a number of possible decay modes that can be explored at the LHC and at other future colliders. In the next section, we summarize the different direct experimental constraints on the charged Higgs states.

\section{DECAY MODES AND EXPERIMENTAL CONSTRAINTS}

We assume the neutral Higgs $H^{0}$ and $A^{0}$ are more massive than the charged Higgs. Among the charged Higgs, $H^{ \pm}$is heavier than $H^{ \pm \pm}$. The doubly charged Higgs boson $H^{ \pm \pm}$of this model can decay into the leptonic or bosonic states and gives unique signatures at high energy colliders. The partial decay widths and branching ratios of the $H^{ \pm \pm}$ depend on the triplet vev $v_{\Delta}$. For the smaller triplet vev, the $H^{ \pm \pm}$predominantly decays into the same-sign leptonic states $H^{ \pm \pm} \rightarrow l^{ \pm} l^{ \pm}$, whereas for larger $v_{\Delta}$, the gauge boson mode $H^{ \pm \pm} \rightarrow W^{ \pm} W^{ \pm}$becomes dominant $[20,22,23]$. The relevant decay widths are calculated to be

$$
\begin{aligned}
\Gamma\left(H^{ \pm \pm} \rightarrow l_{i}^{ \pm} l_{j}^{ \pm}\right)= & \Gamma_{l_{i} l_{j}}=\frac{M_{H}^{ \pm \pm}}{\left(1+\delta_{i j}\right) 8 \pi}\left|\frac{M_{\nu_{i j}}}{v_{\Delta}}\right|^{2}, \\
& M_{\nu}=Y_{\Delta} v_{\Delta}, \\
\Gamma\left(H^{ \pm \pm} \rightarrow W^{ \pm} W^{ \pm}\right) & =\Gamma_{W^{ \pm} W^{ \pm}} \\
& =\frac{g^{2} v_{\Delta}^{2}}{8 \pi M_{H^{ \pm \pm}}} \sqrt{1-\frac{4}{r_{W}^{2}}}\left[\left(2+\left(r_{W} / 2-1\right)^{2}\right)\right] .
\end{aligned}
$$

Here, $M_{\nu}$ denotes the neutrino mass matrix, $i, j$ are the generation indices, and $\Gamma_{l_{i} l_{j}}$ and $\Gamma_{W^{ \pm} W^{ \pm}}$are the partial decay widths for the $H^{ \pm \pm} \rightarrow l_{i}^{ \pm} l_{j}^{ \pm}$and $H^{ \pm \pm} \rightarrow W^{ \pm} W^{ \pm}$ channels, respectively. The parameter $r_{W}$ represents the ratio of $H^{ \pm \pm}$and the $W$ gauge boson masses, $r_{W}=\frac{M_{H^{ \pm \pm}}}{M_{W}}$.

Other than the doubly charged Higgs, the model also contains a singly charged Higgs. The singly charged Higgs $H^{ \pm}$can decay to $l \nu, W Z, W h, t \bar{b}$ final states. Additionally, for nondegenerate charged Higgs masses and triplet vev $v_{\Delta}$ between $10^{-6}$ and $10^{-2} \mathrm{GeV}$, the cascade decay $H^{ \pm} \rightarrow$ $H^{ \pm \pm} W^{*}$ can also become dominant. The partial width for the charged Higgs decaying into $H^{ \pm \pm} W^{-*}$ has the following form:

$\Gamma\left(H^{ \pm} \rightarrow H^{ \pm \pm} W^{-*}\right)=\frac{9 g^{4} M_{H^{ \pm}}}{128 \pi^{3}} \cos ^{2} \beta_{ \pm} G\left(\frac{M_{H^{ \pm \pm}}^{2}}{M_{H^{ \pm}}^{2}}, \frac{M_{W}^{2}}{M_{H^{ \pm}}^{2}}\right)$.

In the above, $\beta_{ \pm}$is the charged Higgs mixing angle. For the expression of the function $G$ and other partial decay widths of $H^{ \pm}$into two fermions mode and two gauge bosons mode; see [26]. We show the branching ratio of $H^{ \pm \pm}$ and $H^{ \pm}$in Fig. 1 for two benchmark values of doubly charged Higgs mass $M_{H^{ \pm \pm}}=247.3 \mathrm{GeV}$ and $M_{H^{ \pm \pm}}=$ $894.02 \mathrm{GeV}$, respectively. In the upper panel of Fig. 1, we show the variation of the branching ratios of the doubly charged Higgs boson for the two chosen benchmark mass points. The lower panel shows the variation of the branching ratio of singly charged Higgs $H^{ \pm}$into different channels. The lower panel has a different response with the increase of the doubly charged Higgs mass, which also implies the increase of the masses of different other charged and neutral Higgs states. From the top panel, this is evident, that there is hardly any change in the branching ratio of doubly charged Higgs for the variation of its mass, except a slight shift in the overlapping region of the two branching ratios. On the other hand, in the lower panel, the scenario is completely different, and one can easily see a huge variation in the branching ratio of the different decay channels of $H^{ \pm}$due to the change in mass of the doubly charged Higgs. This happens because with the increase of the doubly charged Higgs mass, the ratio $\frac{M_{H^{ \pm \pm}}}{M_{H^{ \pm}}} \rightarrow 1$; hence, the decrement in the decay width of $\mathrm{H}^{+} \rightarrow \mathrm{H}^{++} \mathrm{W}^{-*}$ channel occurs due to the phase space suppression.

A number of searches have been proposed at the LHC to discover $H^{ \pm \pm}$using multilepton signatures. The searches in [22-24,47] focused on the pair and associated production with the $H^{ \pm \pm}$decaying into leptonic gauge boson states. Below we discuss the existing constraints on $H^{ \pm \pm}$ from the LEP and LHC searches.

(i) Constraint from the LEP-II: The search for doubly charged Higgs boson $H^{ \pm \pm}$decaying into charged leptons has been performed at the LEP-II. This constrains the mass $M_{H^{ \pm \pm}}>97.3 \mathrm{GeV}$ [50] at $95 \%$ C.L.

(ii) Constraints from pair and associated production: Stringent constraints on $M_{H^{ \pm \pm}}$have been placed by the $13 \mathrm{TeV}$ LHC searches. These searches analyzed the $H^{ \pm \pm} \rightarrow l^{ \pm} l^{ \pm}$channel. The CMS Collaboration looked for different leptonic flavors including $e e, e \mu, e \tau, \mu \mu, \mu \tau$, and $\tau \tau$. In addition, the CMS searches also include the associated production $p p \rightarrow H^{ \pm \pm} H^{\mp}$ and the subsequent decays $H^{ \pm} \rightarrow$ $l^{ \pm} \nu$. This combined channel of pair production and 

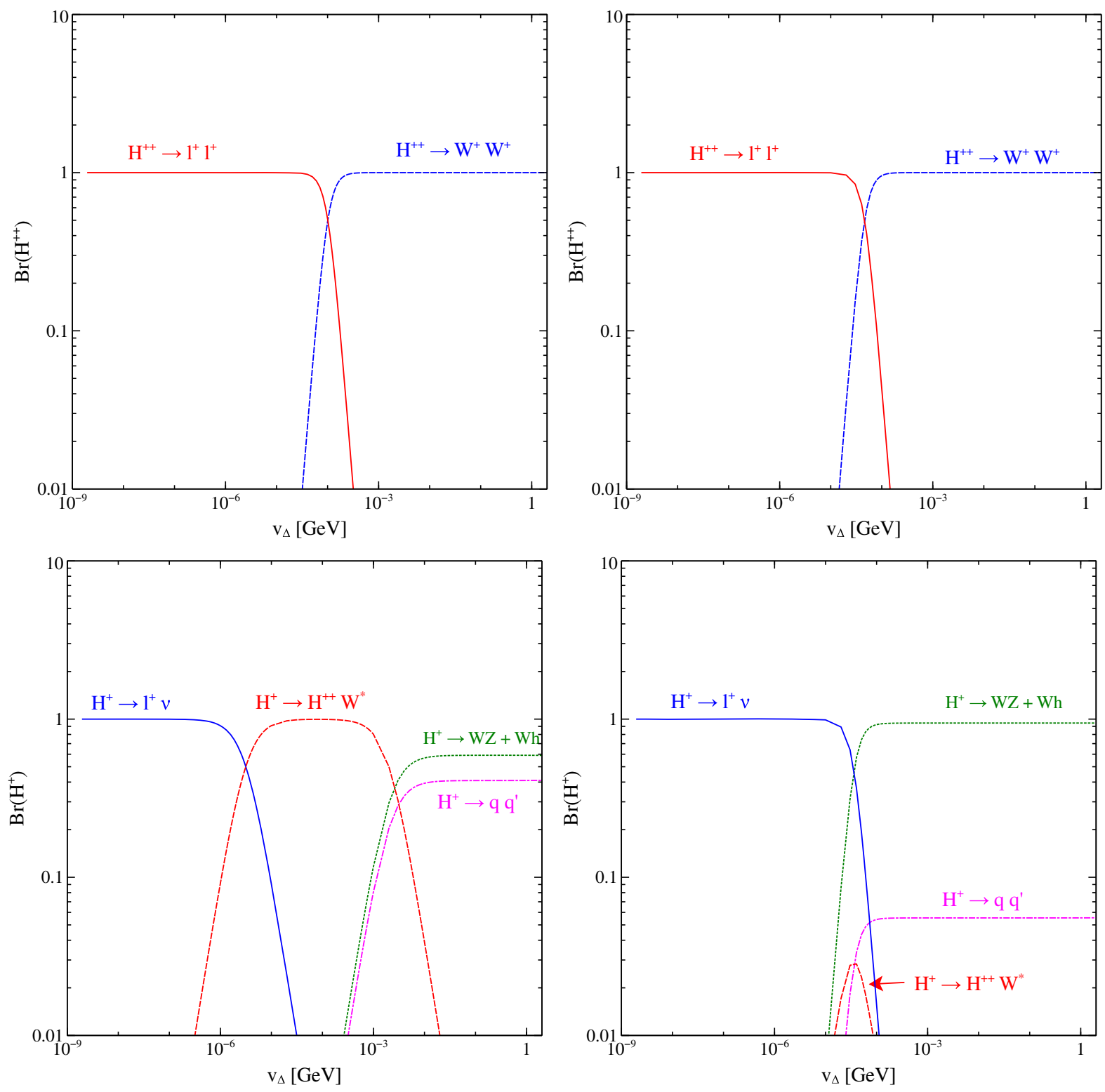

FIG. 1. Upper panels: The branching ratios of $H^{ \pm \pm}$for masses $M_{H^{ \pm \pm}}=247.30 \mathrm{GeV}$ and $M_{H^{ \pm \pm}}=894.02 \mathrm{GeV}$. Lower panels: Branching ratios of $H^{ \pm}$for the masses $M_{H^{ \pm}}=250.35 \mathrm{GeV}$ and $M_{H^{ \pm}}=894.89 \mathrm{GeV}$. The other relevant parameters are kept fixed at $\lambda_{i}=0.1$ (for $i=1$ to 4 ) and $\lambda=0.52$.

associated production gives the stringent constraint $M_{H^{ \pm \pm}}>820 \mathrm{GeV}$ [39] at $95 \%$ C.L. for $e, \mu$ flavor. The realistic bound depends on the neutrino mass matrix [20]. Similar constraints from ATLAS searches have been placed on the mass of doubly charged Higgs that takes into account only pair production. The bound is $M_{H^{ \pm \pm}}>870 \mathrm{GeV}$ at 95\% C.L. [38]. Note that these limits are valid only for a small triplet vev $v_{\Delta}<10^{-4} \mathrm{GeV}$. Additionally, ATLAS looked into the pair production of a doubly charged Higgs with subsequent decays into gauge bosons, resulting in multilepton final states. The search in [40] has constrained the mass of doubly charged Higgs $M_{H^{ \pm \pm}}$between 200 and $220 \mathrm{GeV}$ at 95\% C.L. This is valid for the triplet vev $v_{\Delta}>10^{-4} \mathrm{GeV}$, where the gauge boson decay is most dominant.

(iii) Constraint from vector boson fusion (VBF): For larger values of the triplet vev $v_{\Delta}>10^{-4} \mathrm{GeV}$, the leptonic branching ratio becomes smaller. Instead, the decay mode $H^{ \pm \pm} \rightarrow W^{ \pm} W^{ \pm}$is dominant. Therefore, the searches in VBF become more important. A search for $p p \rightarrow j j H^{ \pm \pm} \rightarrow j j W^{ \pm} W^{ \pm}$at the $8 \mathrm{TeV}$ LHC in the VBF channel sets a constraint on the triplet vev $v_{\Delta} \sim$ $25 \mathrm{GeV}$ for $M_{H^{ \pm \pm}} \sim 300 \mathrm{GeV}$ [41]. This constraint has been updated [42] using $13 \mathrm{TeV}$ data at the LHC. 
Such a large triplet vev is anyway excluded by the $\rho$ parameter bound [19] in the minimal type-II seesaw model.

The abovementioned constraints imply that a large range of the triplet vev $v_{\Delta}>10^{-4} \mathrm{GeV}$ exists, where low mass of $M_{H^{ \pm \pm}}>220 \mathrm{GeV}$ is still allowed. For the lower triplet vev $v_{\Delta}<10^{-4} \mathrm{GeV}$, the mass constraint is more conservative $M_{H^{ \pm \pm}}>870 \mathrm{GeV}$. In our analysis of tetralepton signatures, we therefore choose both the lighter and heavier mass points.

\section{LARGE TRIPLET VEV AND SAME-SIGN TETRALEPTON SIGNATURE FOR $\sqrt{s}=14 \mathrm{TeV}$}

We explore the tetralepton signature arising from a lighter charged Higgs and neutral Higgs decay. We consider associated production of $H^{ \pm}$along with $H^{0} / A^{0}$. For a triplet vev between $10^{-5} \mathrm{GeV}<v_{\Delta}<10^{-3} \mathrm{GeV}$ and assuming mass hierarchy between singly and doubly charged Higgs $M_{H^{ \pm}}>M_{H^{ \pm \pm}}$, the cascade decay of $H^{ \pm}$into $H^{ \pm \pm} W^{*}$ is predominant. In the same triplet vev region, $H^{0} / A^{0} \rightarrow$ $H^{ \pm} W^{*}$ decay is also significantly large. We furthermore consider the gauge boson decay modes of $H^{ \pm \pm} \rightarrow W^{ \pm} W^{ \pm}$ that has a large branching ratio for $v_{\Delta}>10^{-4} \mathrm{GeV}$ and subsequent leptonic decay of the produced on-shell $W^{ \pm}$. For the signal, therefore, the complete process is [56]

(i) $p p \rightarrow H^{ \pm} H^{0} / H^{ \pm} A^{0} \rightarrow H^{ \pm \pm} W^{ \pm *} H^{ \pm} W^{\mp *} \rightarrow$ $H^{ \pm \pm} W^{ \pm *} H^{ \pm \pm} W^{\mp *} W^{\mp *} \rightarrow 4 W^{ \pm}+X$,

(ii) $p p \rightarrow H^{0} A^{0} \rightarrow H^{ \pm} W^{\mp *} H^{ \pm} W^{\mp *} \rightarrow$ $H^{ \pm \pm} W^{ \pm *} H^{ \pm \pm} W^{\mp *} W^{\mp *} W^{\mp *} \rightarrow 4 W^{ \pm}+Y$.

The Feynman diagrams for the above two processes have been shown in Fig. 2. Note that this phenomenon of wrongsign leptons production occurs as $\Delta^{0}$ can oscillate to $\Delta^{0 \dagger}$ and vice versa. As a result, $H^{0}$ and $A^{0}$ sharing the same final states can mix together like in the $B^{0}-\overline{B^{0}}$ system. Finally, we can write the cross section for these signals as

(i) $\sigma\left(p p \rightarrow H^{ \pm} H^{0} / A^{0}\right) \times F_{1} \times \operatorname{Br}\left(H^{ \pm} \rightarrow H^{ \pm \pm} W^{-*}\right)^{2} \times$ $\operatorname{Br}\left(H^{0} / A^{0} \rightarrow H^{ \pm} W^{-*}\right) \times \operatorname{Br}\left(H^{ \pm \pm} \rightarrow W^{ \pm} W^{ \pm}\right)^{2}$

(ii) $\sigma\left(p p \rightarrow H^{0} A^{0}\right) \times F_{2} \times \mathrm{Br}\left(\mathrm{H}^{0} / \mathrm{A}^{0} \rightarrow \mathrm{H}^{ \pm} \mathrm{W}^{-*}\right)^{2} \times$ $\mathrm{Br}\left(\mathrm{H}^{ \pm} \rightarrow \mathrm{H}^{ \pm \pm} \mathrm{W}^{-*}\right) \times \mathrm{Br}\left(\mathrm{H}^{ \pm \pm} \rightarrow \mathrm{W}^{ \pm} \mathrm{W}^{ \pm}\right)^{2}$.

In the above, $F_{1,2}$ are

$$
\begin{gathered}
F_{1}=\frac{x^{2}}{1+x^{2}}, \quad F_{2}=\frac{2+x^{2}}{2\left(1+x^{2}\right)} \times \frac{x^{2}}{2\left(1+x^{2}\right)}, \\
\text { with } \quad x=\frac{\delta M}{\Gamma_{H^{0} / A^{0}}}=\frac{M_{H^{0}}-M_{A^{0}}}{\Gamma_{H^{0} / A^{0}}}
\end{gathered}
$$
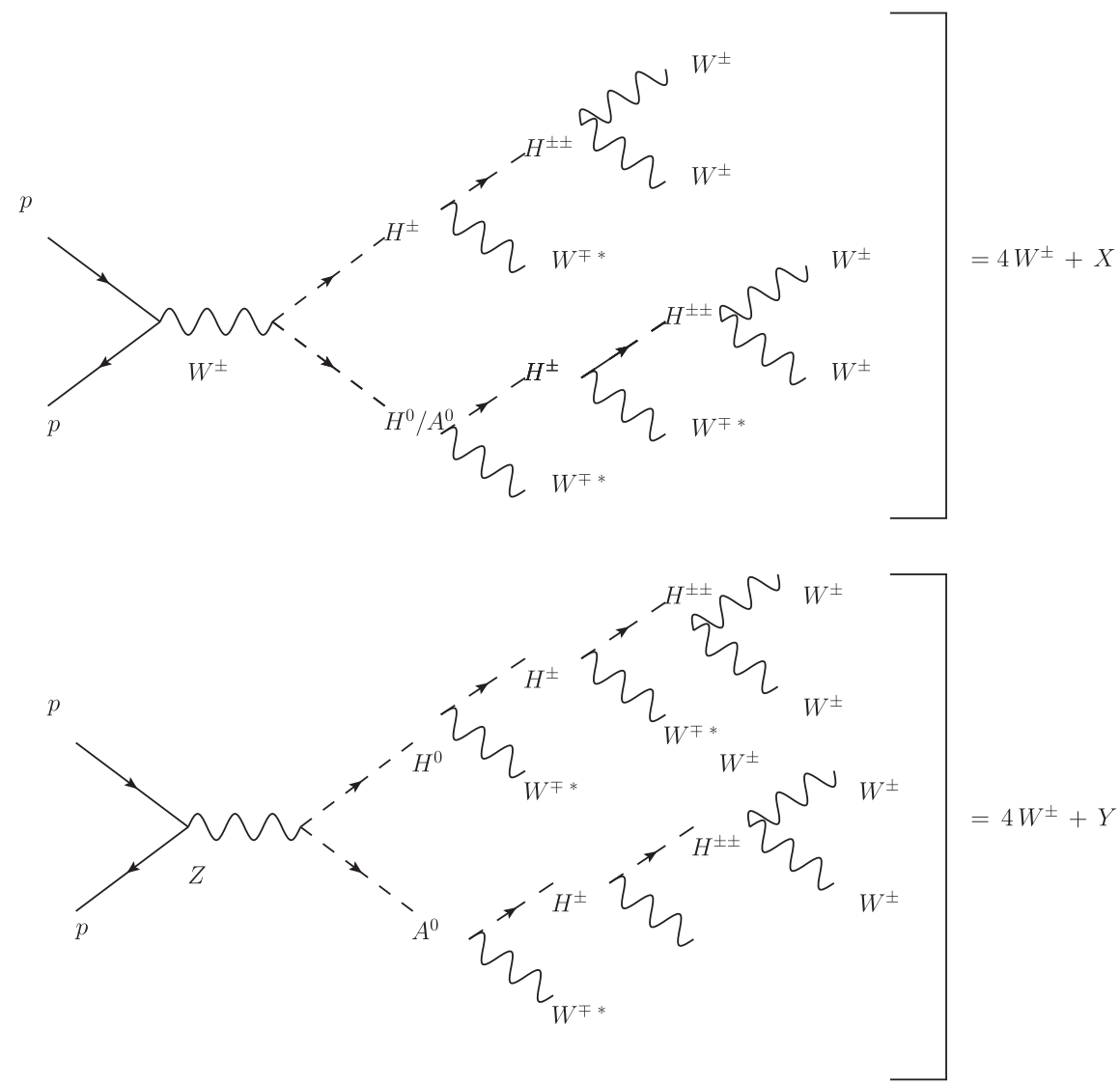

FIG. 2. Feynman diagrams for $p p \rightarrow H^{ \pm} H^{0} / A^{0}, p p \rightarrow H^{0} A^{0}$, and the subsequent decays of $H^{0} / A^{0} \rightarrow H^{ \pm} W^{-*}, H^{ \pm} \rightarrow H^{ \pm \pm} W^{-*}$, and $H^{ \pm \pm} \rightarrow W^{ \pm} W^{ \pm}$. 
when the two decay widths $\Gamma_{H^{0}}$ and $\Gamma_{A^{0}}$ are nearly equal, i.e., $\Gamma_{H^{0}} \simeq \Gamma_{A^{0}}$. The generalization of these two processes to the case of $\Gamma_{A^{0}} \neq \Gamma_{H^{0}}$ is

(i) $\sigma\left(p p \rightarrow H^{ \pm} H^{0} / A^{0}\right) \times G_{1} \times \mathrm{Br}\left(\mathrm{H}^{ \pm} \rightarrow \mathrm{H}^{ \pm \pm} \mathrm{W}^{-*}\right)^{2} \times$ $\mathrm{Br}\left(\mathrm{H}^{0} / \mathrm{A}^{0} \rightarrow \mathrm{H}^{ \pm} \mathrm{W}^{-*}\right) \times \mathrm{Br}\left(\mathrm{H}^{ \pm \pm} \rightarrow \mathrm{W}^{ \pm} \mathrm{W}^{ \pm}\right)^{2}$

(ii) $\sigma\left(p p \rightarrow H^{0} A^{0}\right) \times G_{2} \times \mathrm{Br}\left(\mathrm{H}^{0} / \mathrm{A}^{0} \rightarrow \mathrm{H}^{ \pm} \mathrm{W}^{-*}\right)^{2} \times$ $\mathrm{Br}\left(\mathrm{H}^{ \pm} \rightarrow \mathrm{H}^{ \pm \pm} \mathrm{W}^{-*}\right) \times \mathrm{Br}\left(\mathrm{H}^{ \pm \pm} \rightarrow \mathrm{W}^{ \pm} \mathrm{W}^{ \pm}\right)^{2}$,

where $G_{1}$ and $G_{2}$ have the following forms:

$$
\begin{aligned}
G_{1}= & \frac{x^{2}+y^{2}}{2\left(1+x^{2}\right)}, \quad G_{2}=\frac{2+x^{2}-y^{2}}{2\left(1+x^{2}\right)} \times \frac{x^{2}+y^{2}}{2\left(1+x^{2}\right)}, \\
& \text { with } \quad x=\frac{\delta M}{\Gamma}, \\
\Gamma= & \frac{\Gamma_{H}^{0}+\Gamma_{A}^{0}}{2}, \quad \text { and } \quad y=\frac{\Gamma_{H}^{0}-\Gamma_{A}^{0}}{\Gamma_{H}^{0}+\Gamma_{A}^{0}} .
\end{aligned}
$$

Note that, to compute the tetralepton signature, one needs to take into account the leptonic branching ratios from $W$. In our analysis, we consider both the $W \rightarrow l \nu$ with $l=e, \mu$ as well as $W \rightarrow \tau \nu$ with the leptonic decays of $\tau$ included. To compute the cross section, we implement the model in FeynRules (v2.3) [57]. The Universal FeynRules Output (UFO) is then fed into MadGraph5_aMC@NLO (v2.6) [58] that generates the parton-level events. We use the default parton distribution function NNPDF23LO1 [59] for computation. We perform parton showering and hadronization with PYTHIA8 [60] and analyze the HepMC [61] event files. The above cross sections $p p \rightarrow H^{ \pm} H^{0}$ and $p p \rightarrow H^{0} A^{0}$ depend on the masses of the neutral and charged Higgs. We therefore show the variation of associated production cross section of $p p \rightarrow H^{ \pm} H^{0} / A^{0}$ and

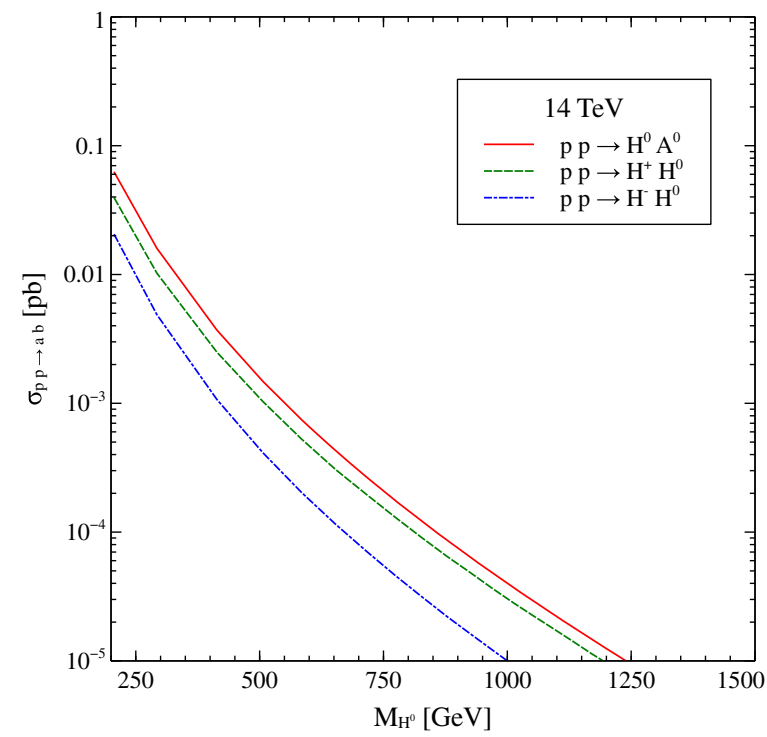

$p p \rightarrow H^{0} A^{0}$ with the mass of $H^{0}$ in Fig. 3. For c.m. energy $\sqrt{s}=14 \mathrm{TeV}$, the cross section for $p p \rightarrow H^{0} A^{0}$ varies between 1 and $70 \mathrm{fb}$ for neutral Higgs mass between 200 and $500 \mathrm{GeV}$. For $p p \rightarrow H^{+} H^{0} / A^{0}$, the cross section is very similar, only lower by a factor of $\mathcal{O}(1.5)$. For $p p \rightarrow H^{-} H^{0} / A^{0}$, the cross section is smaller due to the parton distribution function. In addition, we also show the production cross section for a future $p p$ collider with c.m. energy $\sqrt{s}=100 \mathrm{TeV}$. As is evident from the right panel of Fig. 3, the production cross section is quite large for higher c.m. energy, and multi-TeV Higgs mass can be probed.

Note that the production cross sections for $p p \rightarrow$ $H^{ \pm} H^{0} / A^{0}$ depend on both the parameters $\lambda_{4}$ and the triplet vev $v_{\Delta}$. For a fixed value of $\mu$, the triplet vev primarily governs the masses of the Higgs $H^{ \pm}, H^{0} / A^{0}$, while the parameter $\lambda_{4}$ determines their mass difference. In the left panel of Fig. 4, we show the variation of the production cross section for the process $p p \rightarrow H^{+} H^{0}$ in the $v_{\Delta}-\lambda_{4}$ plane for a benchmark value of neutral Higgs, $M_{H^{0}} \sim$ $253 \mathrm{GeV}$. For the process $p p \rightarrow H^{-} H^{0}$, the plot is very similar; only the production cross section is relatively smaller by a factor of 2 . The channel $p p \rightarrow H^{0} A^{0}$ has the largest cross section, larger than $p p \rightarrow H^{+} H^{0}$ by almost a factor of $\mathcal{O}(1.4-1.7)$. Since $\lambda_{4}$ has a very nominal effect on the mass splitting of $H^{0}, A^{0}$, the cross section of this channel is almost fixed in the entire plane of $\lambda_{4}-v_{\Delta}$, and thus does not vary.

The doubly, singly charged, and neutral Higgs bosons will decay through a number of subsequent decay modes, leading to the same-sign tetralepton final states. The two key parameters are again the triplet vev $v_{\Delta}$ and

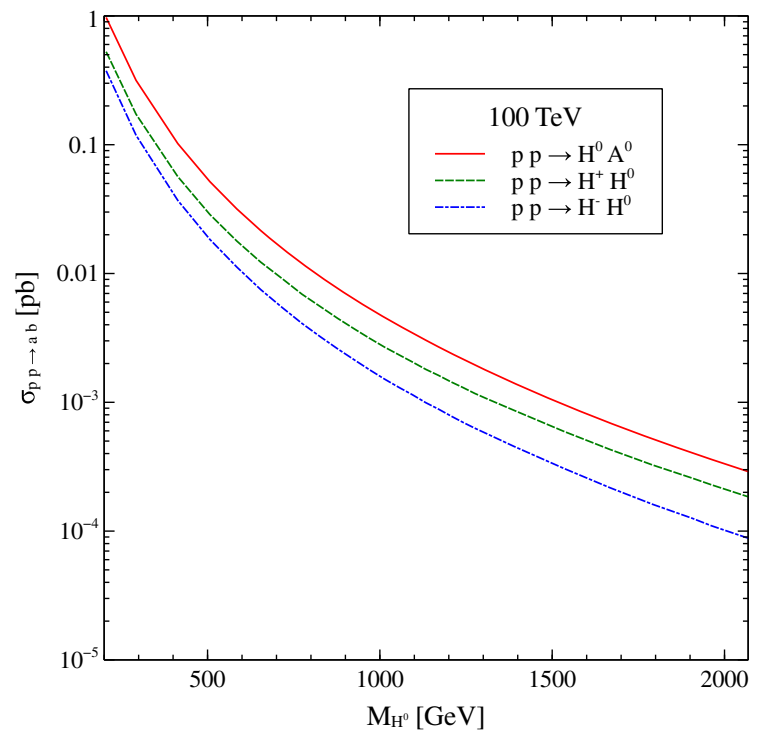

FIG. 3. Left panel: Cross section for associated production $H^{ \pm} H^{0}, H^{0} A^{0}$ vs the mass of $H^{ \pm}$. The c.m. energy is $\sqrt{s}=14 \mathrm{TeV}$. The other parameters are fixed at $\lambda_{i}=0.1$ ( $i=1$ to 4 ), $\lambda=0.52, v_{\Delta}=10^{-3} \mathrm{GeV}$, and $\mu$ has been varied between $2 \times 10^{-3} \mathrm{GeV}$ and $4.5 \times 10^{-2} \mathrm{GeV}$ to vary the mass of the particles. The production cross sections for $H^{+} A^{0}$ and $H^{-} A^{0}$ are same as $H^{+} H^{0}$ and $H^{-} H^{0}$, respectively. Right panel: The same plot for higher c.m. energy $\sqrt{s}=100 \mathrm{TeV}$. 

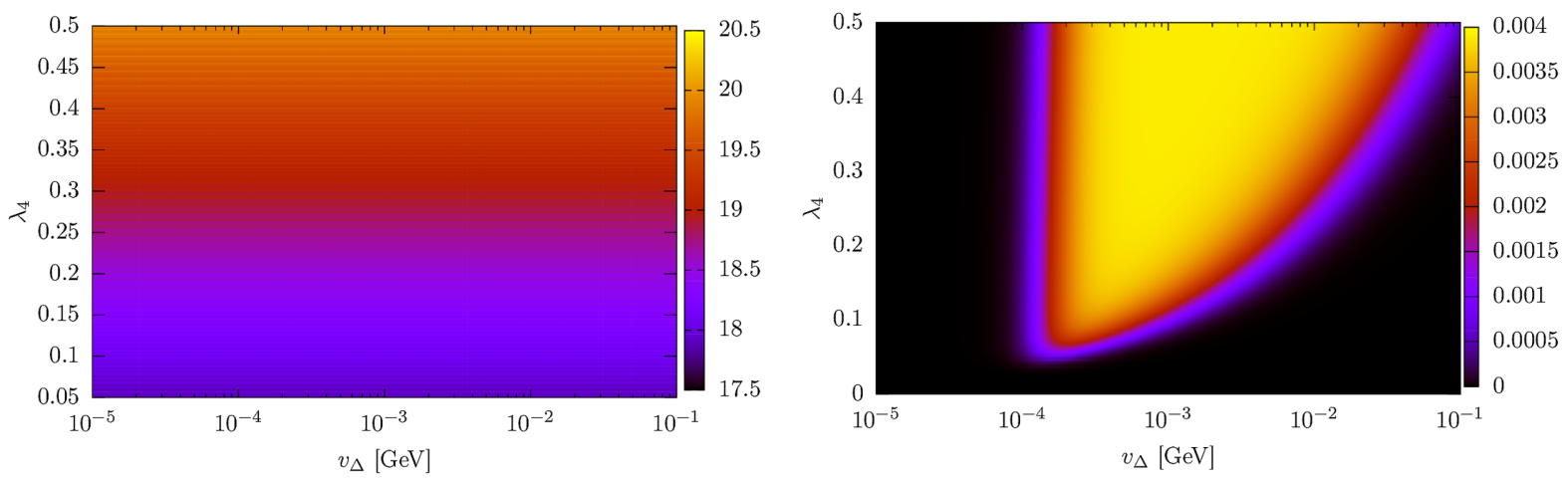

FIG. 4. Left panel: Cross section in fb for the channel $p p \rightarrow H^{+} H^{0}$ for the c.m. energy $\sqrt{s}=14 \mathrm{TeV}\left(M_{A^{0}}=253 \mathrm{GeV}\right)$. Right panel: Product of branching ratios $\operatorname{Br}\left(H^{ \pm} \rightarrow H^{ \pm \pm} W^{-*}\right)^{2} \times \operatorname{Br}\left(H^{0} / A^{0} \rightarrow H^{ \pm} W^{-*}\right) \times \operatorname{Br}\left(H^{ \pm \pm} \rightarrow W^{ \pm} W^{ \pm}\right)^{2} \times \operatorname{Br}\left(W^{ \pm} \rightarrow \ell \nu\right)^{4}$ for the process $p p \rightarrow H^{ \pm} H^{0} / A^{0}$ with mass of $A^{0}$ being fixed as $M_{A^{0}}=253 \mathrm{GeV}$. For the second process $p p \rightarrow H^{0} / A^{0}$, the behavior of the product of the branching ratio will be the same.

the coupling $\lambda_{4}$. Since a number of branching ratios are involved in the same-sign tetralepton process, we show the product of these branching ratios. In the right panel of Fig. 4, we show the variation of the product of branching ratios $\operatorname{Br}\left(H^{ \pm} \rightarrow H^{ \pm \pm} W^{-*}\right)^{2} \times \operatorname{Br}\left(H^{0} / A^{0} \rightarrow H^{ \pm} W^{-*}\right) \times$ $\operatorname{Br}\left(H^{ \pm \pm} \rightarrow W^{ \pm} W^{ \pm}\right)^{2} \times \operatorname{Br}\left(W^{ \pm} \rightarrow \ell \nu\right)^{4}$ for the process

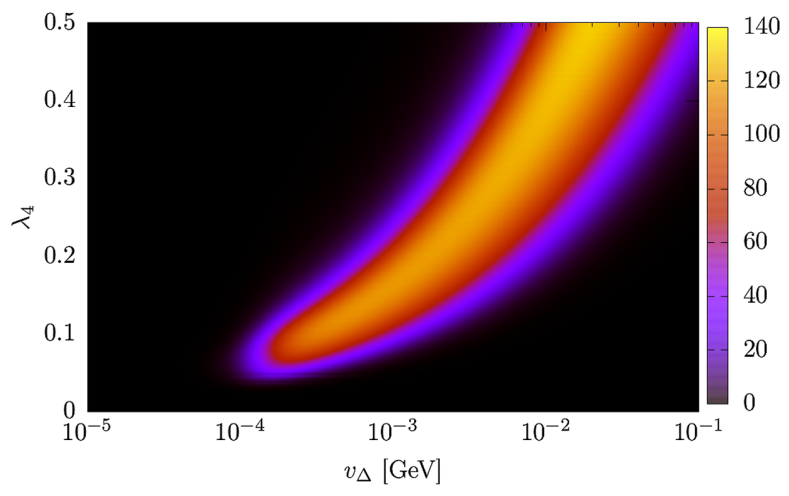

$p p \rightarrow H^{ \pm} H^{0} / A^{0}$ in the $v_{\Delta}-\lambda_{4}$ plane. From the top panel of Fig. 1, it is evident that the doubly charged Higgs $H^{ \pm \pm}$ decays predominantly to the same-sign $W^{ \pm} W^{ \pm}$state. For a smaller range of the triplet vev, it entirely decays to the $l^{ \pm} l^{ \pm}$ final state. This is reflected in Fig. 4, where there is a sharp change in the branching ratio around $10^{-4} \mathrm{GeV}$.

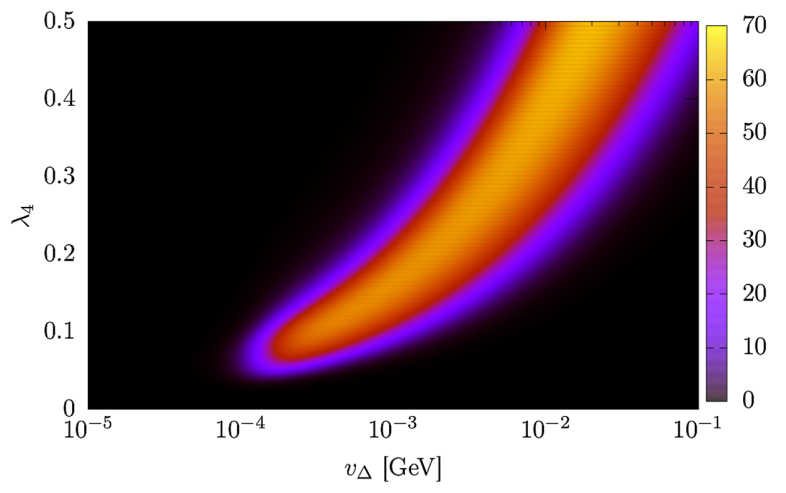

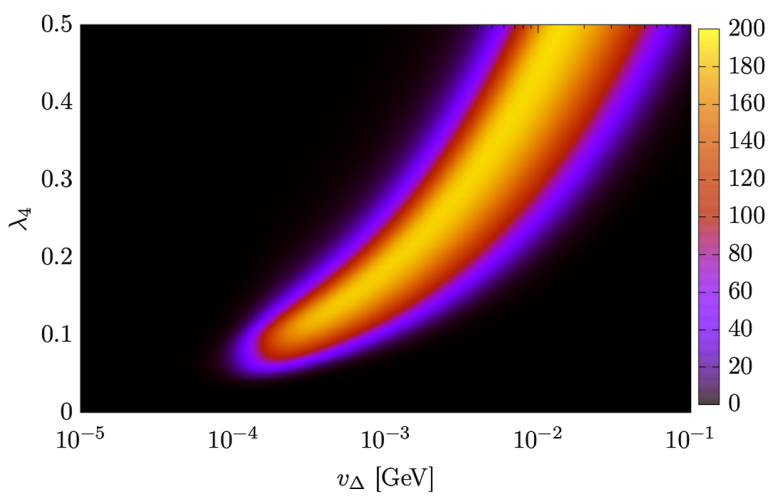

FIG. 5. This plot represents the number of same-sign tetralepton events for mass $M_{H^{0}} \sim M_{A^{0}}=253 \mathrm{GeV}$. Left figure of upper panel: Number of same-sign tetralepton events $\ell^{+} \ell^{+} \ell^{+} \ell^{+}+X$ from $p p \rightarrow H^{+} H^{0} / A^{0}$ followed by subsequent decays of $H^{+}, H^{0}, A^{0}$. Right figure of upper panel: Number of same-sign tetralepton events $\ell^{-} \ell^{-} \ell^{-} \ell^{-}+X$ from $p p \rightarrow H^{-} H^{0} / A^{0}$ and subsequent decays. Lower panel: Number of same-sign tetralepton events $\ell^{+} \ell^{+} \ell^{+} \ell^{+}+Y$ or $\ell^{-} \ell^{-} \ell^{-} \ell^{-}+Y$ from $p p \rightarrow H^{0} A^{0}$ and subsequent decays. For the doubly charged Higgs, we consider the $H^{ \pm \pm} \rightarrow W^{ \pm} W^{ \pm}$decay mode. The c.m. energy $\sqrt{s}=14$ TeV and we consider luminosity $\mathcal{L}=3000 \mathrm{fb}^{-1}$. For this range of $\lambda_{4}$, the masses of $H^{ \pm}$and $H^{ \pm \pm}$vary at most by $M_{H^{0}}-M_{H^{++}} \sim 32 \mathrm{GeV}$ and $M_{H^{0}}-M_{H^{+}} \sim 15 \mathrm{GeV}$, respectively. 


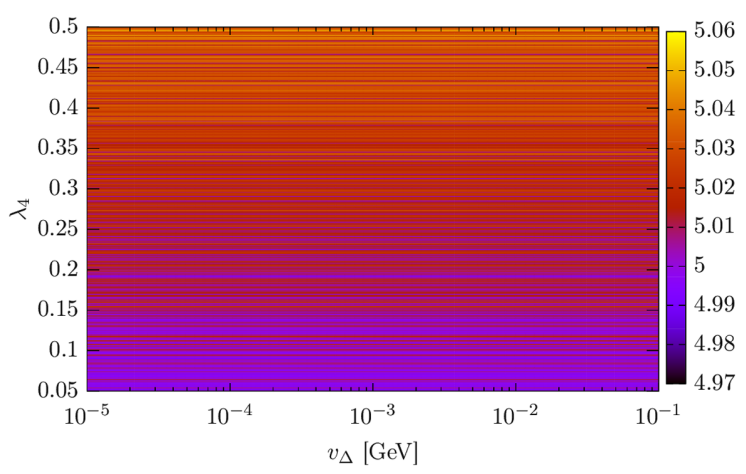

FIG. 6. Cross section in $\mathrm{fb}$ for the channel $p p \rightarrow H^{+} H^{0}$ for the mass $M_{A^{0}}=900 \mathrm{GeV}$. We consider c.m. energy $\sqrt{s}=100 \mathrm{TeV}$.

The product goes to zero on the left side of this line (as shown by the black region). On the right side of this line, the product can be large, as indicated by the color bar. We stress that the product of the branching ratios has a significantly large value for a wide range of the triplet vev $10^{-4} \mathrm{GeV}<v_{\Delta}<10^{-2} \mathrm{GeV}$. Therefore, in this region, there will be handful of events for same-sign tetralepton final states that can be tested at the LHC. In the next section, we will see how this large range of the triplet vev shrinks to a very narrow range for higher masses of the charged and neutral Higgs. This occurs due to significant change in branching ratios of the channel $H^{ \pm} \rightarrow H^{ \pm \pm} W^{-*}$ for the same value of $\lambda_{4}$.

In Fig. 5, we show the variation of the number of events for the same-sign tetralepton signature, where we consider integrated luminosity $\mathcal{L}=3000 \mathrm{fb}^{-1}$. This has been obtained by folding the production cross section with the overall branching ratio and integrated luminosity. We also implement a few basic cuts at the PYTHIA level. These are $p_{T}\left(e^{ \pm} / \mu^{ \pm}\right)>10 \mathrm{GeV},\left|\eta\left(e^{ \pm} / \mu^{ \pm}\right)\right|<2.5$. We obtain a cut efficiency $c_{\text {eff }}=0.62$ for $M_{H^{0}}=253 \mathrm{GeV}$ that we include in our calculation of total number of events. We consider the processes $p p \rightarrow H^{+} H^{0} / A^{0}$ (top left), $p p \rightarrow$ $H^{-} H^{0} / A^{0}$ (top right), and $p p \rightarrow H^{0} A^{0}$ (bottom). To calculate the number of events, we followed the prescription given at the beginning of Sec. IV. As we can see from the bottom left plot of Fig. 1, for the low mass range of the particle spectrum, the channel $H^{ \pm} \rightarrow H^{ \pm \pm} W^{-*}$ has a $100 \%$ branching ratio for a wide range of the triplet vev. Hence, in all three plots, we get a reasonable number of events for the triplet vev $v_{\Delta} \sim 10^{-4}-10^{-1} \mathrm{GeV}$. As exhibited in Fig. 3, the cross section for the different final states has the following hierarchies $\sigma\left(p p \rightarrow H^{0} A^{0}\right)>p p \rightarrow \sigma\left(H^{+} H^{0} / A^{0}\right)>$ $\sigma\left(p p \rightarrow H^{-} H^{0} / A^{0}\right)$. The same hierarchy also translates in the number of events. All three plots have a similar kind of morphology in the $v_{\Delta}-\lambda_{4}$ plane, and the nature of the
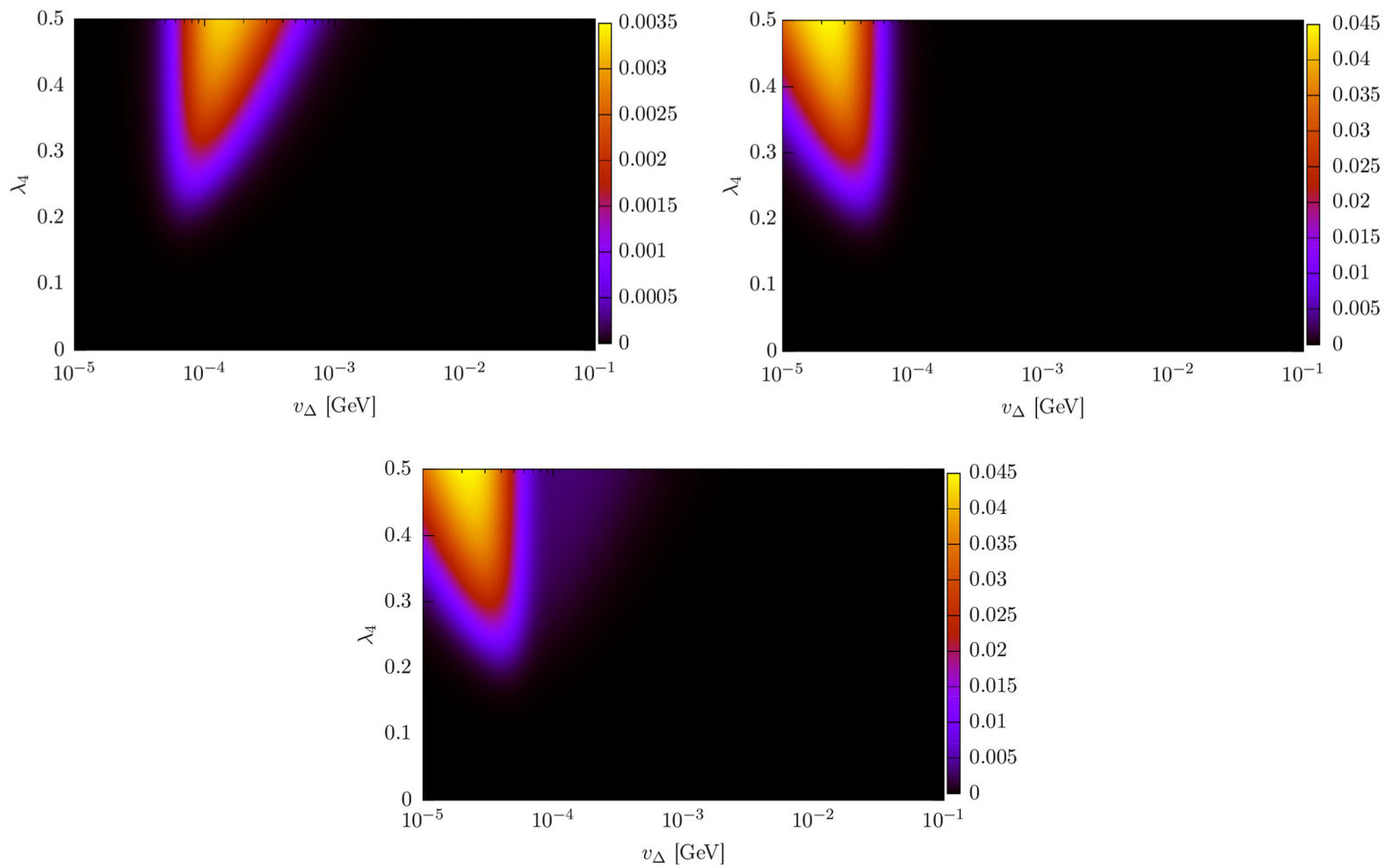

FIG. 7. Upper panels: This represents the product of branching ratios $\operatorname{Br}\left(H^{ \pm} \rightarrow H^{ \pm \pm} W^{-*}\right)^{2} \times \operatorname{Br}\left(H^{0} / A^{0} \rightarrow H^{ \pm} W^{-*}\right) \times \operatorname{Br}\left(H^{ \pm \pm} \rightarrow\right.$ $\left.W^{ \pm} W^{ \pm}\right)^{2} \times \operatorname{Br}\left(W^{ \pm} \rightarrow \ell \nu\right)^{4}$ (left figure), $\operatorname{Br}\left(H^{ \pm} \rightarrow H^{ \pm \pm} W^{-*}\right)^{2} \times \operatorname{Br}\left(H^{0} / A^{0} \rightarrow H^{ \pm} W^{-*}\right) \times \operatorname{Br}\left(H^{ \pm \pm} \rightarrow \ell^{ \pm} \ell^{ \pm}\right)^{2}$ (right figure). Lower panel: The sum of these two products of branching ratios for the process $p p \rightarrow H^{ \pm} H^{0} / A^{0}$ with mass of $A^{0}$ being fixed as $M_{A^{0}}=900 \mathrm{GeV}$. For the process $p p \rightarrow H^{0} A^{0}$, the product of the branching ratio is very similar. 
variation of the number of events can be understood in the following way. Since we are considering the $H^{ \pm \pm} \rightarrow W^{ \pm} W^{ \pm}$ channel, which starts contributing when the triplet vev is $v_{\Delta}>10^{-4} \mathrm{GeV}$, the number of events $N_{\text {evt }}>5$ starts around this region of the triplet vev. As shown in Fig. 4, the cross section increases with larger $\lambda_{4}$, while the branching ratio for the channel $H^{ \pm} \rightarrow H^{ \pm \pm} W^{-*}$ decreases (bottom right plot of Fig. 1) for the larger triplet vev, leading to the specific variation of the number of events shown in Fig. 5.

\section{INCLUSIVE SAME-SIGN TETRALEPTON SIGNATURE FOR $\sqrt{s}=100 \mathrm{TeV}$}

We consider a heavier Higgs and analyze its discovery prospect at a future $p p$ collider that can operate with c.m. energy $\sqrt{s}=100 \mathrm{TeV}$. Because of the suppression from a number of branching ratios, the observation of same-sign tetralepton final states will be beyond the scope of $13 \mathrm{TeV}$ LHC. However, this can easily be observed in a future collider with higher c.m. energy. As a benchmark sample, we consider neutral Higgs mass $M_{H^{0} / A^{0}}=900 \mathrm{GeV}$ and variation of the doubly charged Higgs of mass at most by $5 \mathrm{GeV}$ from $M_{H^{0} / A^{0}}$. The chosen value of the doubly charged Higgs mass is consistent with the constraints from

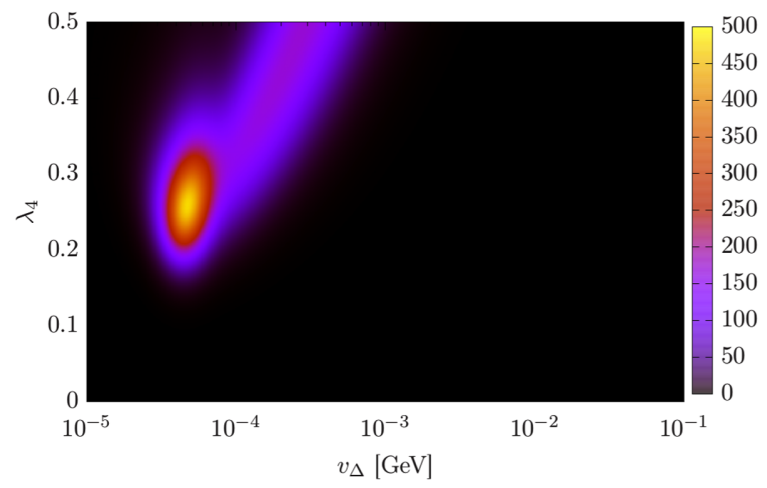

$13 \mathrm{TeV}$ LHC searches for the entire range of the triplet vev $v_{\Delta} \sim 10^{-9}-1 \mathrm{GeV}$. Near the triplet vev $v_{\Delta} \sim 10^{-4}$, both the dilepton and gauge boson modes will substantially contribute. We therefore cover a large range of the triplet vev $v_{\Delta}$ and consider the doubly charged Higgs decaying into both the same-sign dilepton and gauge boson modes. Hence, in addition to the gauge bosons discussed in Sec. IV, the total cross section also contains the following contribution from the dilepton decay mode:

(i) $\sigma\left(p p \rightarrow H^{ \pm} H^{0} / A^{0}\right) \times G_{1} \times \mathrm{Br}\left(\mathrm{H}^{ \pm} \rightarrow \mathrm{H}^{ \pm \pm} \mathrm{W}^{-*}\right)^{2} \times$ $\operatorname{Br}\left(\mathrm{H}^{0} / \mathrm{A}^{0} \rightarrow \mathrm{H}^{ \pm} \mathrm{W}^{-*}\right) \times \mathrm{Br}\left(\mathrm{H}^{ \pm \pm} \rightarrow \ell^{ \pm} \ell^{ \pm}\right)^{2}$

(ii) $\sigma\left(p p \rightarrow H^{0} A^{0}\right) \times G_{2} \times \mathrm{Br}\left(\mathrm{H}^{0} / \mathrm{A}^{0} \rightarrow \mathrm{H}^{ \pm} \mathrm{W}^{-*}\right)^{2} \times$ $\mathrm{Br}\left(\mathrm{H}^{ \pm} \rightarrow \mathrm{H}^{ \pm \pm} \mathrm{W}^{-*}\right)^{2} \times \mathrm{Br}\left(\mathrm{H}^{ \pm \pm} \rightarrow \ell^{ \pm} \ell^{ \pm}\right)^{2}$.

In the above, $l=e, \mu, \tau$, and we finally consider the leptonic branching ratios of $\tau$, while calculating the number of events. The functions $G_{1,2}$ have been described in Sec. IV. We show the variation of the cross section in Fig. 6. The cross section for the mass $M_{A^{0}}=900 \mathrm{GeV}$ varies around $5 \mathrm{fb}$. We next show the variation of the product of branching ratios in Fig. 7 for heavier charged and neutral Higgs. For the triplet vev smaller than $v_{\Delta}<10^{-4} \mathrm{GeV}$, the doubly charged Higgs $H^{ \pm \pm} \rightarrow l^{ \pm} l^{ \pm}$ is dominant, while around $10^{-4} \mathrm{GeV}$, both the gauge boson mode and dilepton are dominant. For a heavier singly

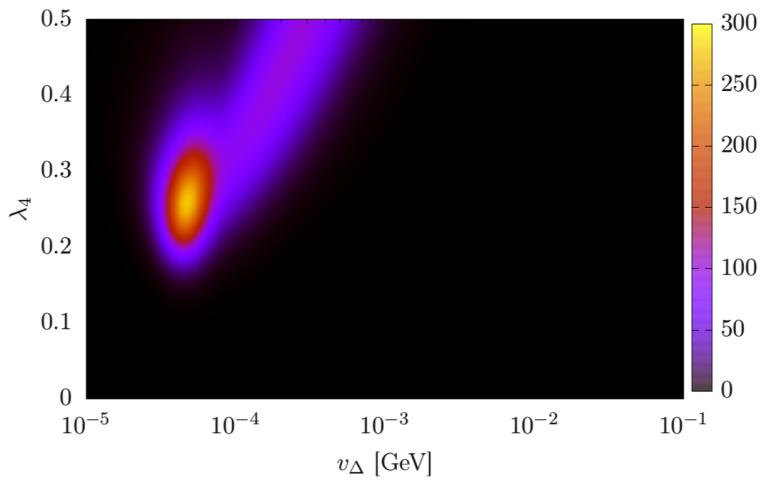

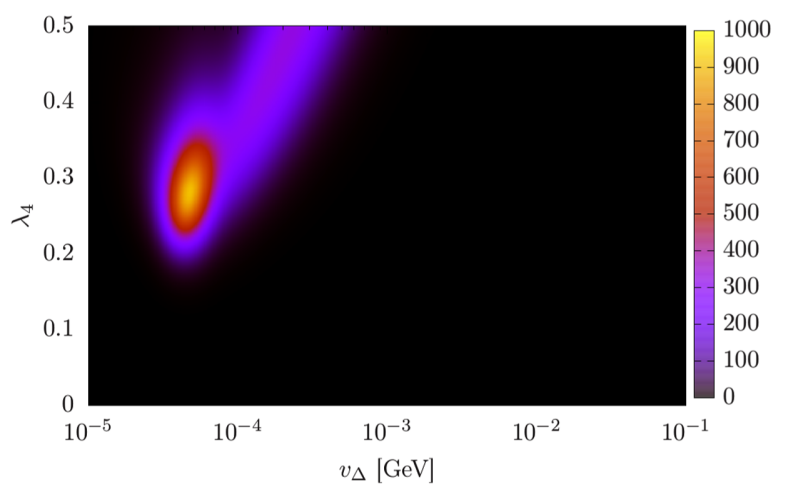

FIG. 8. These figures represent the number of events for mass $M_{A^{0}} / M_{H^{0}}=900 \mathrm{GeV}$. Upper panels: Number of same-sign tetralepton events $\ell^{+} \ell^{+} \ell^{+} \ell^{+}+X$ from $p p \rightarrow H^{+} H^{0} / A^{0}$ and subsequent decays (left) and number of same-sign tetralepton events $\ell^{-} \ell^{-} \ell^{-} \ell^{-}+$ $X$ from $p p \rightarrow H^{-} H^{0} / A^{0}$ and subsequent decays (right). Lower panel: Number of same-sign tetralepton events $\ell^{+} \ell^{+} \ell^{+} \ell^{+}+Y$ or $\ell^{-} \ell^{-} \ell^{-} \ell^{-}+Y$ from $p p \rightarrow H^{0} A^{0}$ and subsequent decays. We consider luminosity $\mathcal{L}=30 \mathrm{ab}^{-1}$. For this range of $\lambda_{4}$, the masses of $H^{ \pm}$ and $H^{ \pm \pm}$vary at most by $M_{H^{0}}-M_{H^{ \pm \pm}} \sim 8.4 \mathrm{GeV}$ and $M_{H^{0}}-M_{H^{ \pm}} \sim 4.2 \mathrm{GeV}$, respectively. 

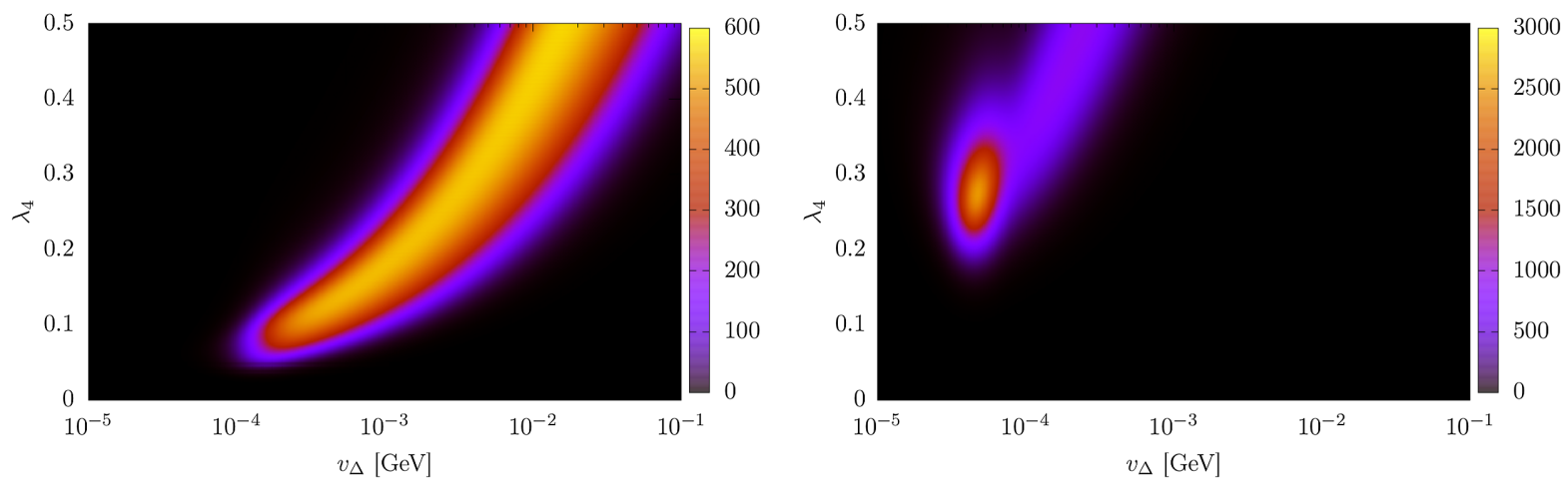

FIG. 9. Total number of same-sign tetralepton events $\left(\ell^{+} \ell^{+} \ell^{+} \ell^{+}+\ell^{-} \ell^{-} \ell^{-} \ell^{-}\right)$for both the cases of $M_{A}^{0}=253 \mathrm{GeV}$ (left figure) and $M_{A^{0}}=900 \mathrm{GeV}$ (right figure).

charged Higgs, the branching ratio for the $H^{ \pm} \rightarrow H^{ \pm \pm} W^{-*}$ decay channel is large for a large value of $\lambda_{4}$. Note that, for $\lambda_{4} \sim 0.1$, the branching ratio becomes more than $1 \%$ in a very small range of the triplet vev (see Fig. 1). This, in turn, has a large effect on the total branching ratio and is clearly visible in all three plots of Fig. 7. The region in $v_{\Delta}$, in which the overall branching ratio is larger than $0.5 \%$ is now considerably smaller. The left plot in the top panel represents the overall branching ratio with only the $H^{ \pm \pm} \rightarrow W^{ \pm} W^{ \pm}$ decay included. The plot in the right panel shows the total branching ratio for $H^{ \pm \pm} \rightarrow l^{ \pm} l^{ \pm}$. The product of the branching ratio is smaller for the case of $H^{ \pm \pm} \rightarrow W^{ \pm} W^{ \pm}$ due to additional suppression from $\operatorname{Br}\left(W^{ \pm} \rightarrow \ell^{ \pm} \nu\right)^{4}$. In the lower panel, we show the sum of these two branching ratios. The higher values of the product of the branching ratios is governed by $H^{ \pm \pm} \rightarrow l^{ \pm} l^{ \pm}$decay mode (relevant for $v_{\Delta} \lesssim 10^{-4} \mathrm{GeV}$ ). More explicitly, we show the $H^{ \pm \pm} \rightarrow$ $W^{ \pm} W^{ \pm}$dominated branching ratio by the blueish region and the $H^{ \pm \pm} \rightarrow l^{ \pm} l^{ \pm}$dominated branching ratio by yellowish region. The total cross section has been computed by folding the branching ratios with the cross section shown in Fig. 6. In addition, we also include a few preliminary cuts, $p_{T}\left(e^{ \pm} / \mu^{ \pm}\right)>10 \mathrm{GeV}, \quad\left|\eta\left(e^{ \pm} / \mu^{ \pm}\right)\right|<2.5$. For $M_{H^{0}}=$ $900 \mathrm{GeV}$ and neutrino oscillation parameters to their best fit values [1], we obtain the cut efficiencies $c_{\text {eff }}=0.64$ in the $H^{ \pm \pm} \rightarrow l^{ \pm} l^{ \pm}$mode and $c_{\text {eff }}=0.62$ in the $H^{ \pm \pm} \rightarrow W^{ \pm} W^{ \pm}$ mode that have been included in this analysis.

In Fig. 8, we present the number of events for heavier doubly charged Higgs and charged and neutral Higgs $(\sim 900 \mathrm{GeV})$. In all three plots, which correspond to the $p p \rightarrow H^{+} H^{0} / A^{0}, p p \rightarrow H^{-} H^{0} / A^{0}$, and $p p \rightarrow H^{0} A^{0}$ processes, its possible to achieve a significantly large number of events in a very narrow region indicated by the yellow patch. This is contrary to the low mass range discussed in the previous section, where we get a reasonable number of events for a wider range of the triplet vev. Figure 9 represents the variation of the total number of events for tetraleptons with either +ve or -ve sign of the leptons. The left panel shows the variation of the sum of the number of events $\left(l^{+} l^{+} l^{+} l^{+}+l^{-} l^{-} l^{-} l^{-}\right)$for the low mass of the particles, and its shape is exactly similar to what we discussed before (see Fig. 5). The figure in the right panel shows the events for higher mass and also has a similar shape to that displayed in Fig. 8.

\section{CONCLUSION}

The type-II seesaw model is one of the most simplest models of neutrino mass generation, where the model is extended by an additional triplet scalar field. Because of an extended Higgs sector and mixing between the SM doublet scalar field and triplet scalar, the model contains few additional Higgs fields, including doubly charged and singly charged Higgs fields, as well as $C P$-even and -odd neutral Higgs fields. In this work, we consider a type-II seesaw model for neutrino mass generation and analyze a unique same-sign tetralepton signature at $p p$ colliders. This arises from the associated production of Higgs fields $H^{ \pm} H^{0}, H^{0} A^{0}$, and the subsequent decay of the neutral Higgs field into a singly charged Higgs state, and the decay of a singly charged field into a doubly charged Higgs state. More precisely, for nondegenerate Higgs masses and for an intermediate triplet vev $v_{\Delta}$ between $10^{-5} \mathrm{GeV}<v_{\Delta}<10^{-2} \mathrm{GeV}$, the neutral and charged Higgs $H^{0}, A^{0}, H^{ \pm}$decay predominantly to $H^{0} / A^{0} \rightarrow H^{ \pm} W^{*}$ and $H^{ \pm} \rightarrow H^{ \pm \pm} W^{*}$. The subsequent decays of $H^{ \pm \pm}$either to same-sign dileptons or to samesign gauge bosons lead to the same-sign tetralepton final states. We analyze this signature for a $p p$ collider, taking into account two different c.m. energies $\sqrt{s}=14 \mathrm{TeV}$ and $\sqrt{s}=100 \mathrm{TeV}$. In our analysis, we choose those benchmark mass points that are consistent with the present limits from the $13 \mathrm{TeV}$ LHC. In particular, for the lower c.m. energy, we explore the tetralepton signatures from a lighter Higgs state, and for a higher c.m. energy, we consider a heavier Higgs states.

As an illustrative example, we first consider a large triplet vev $v_{\Delta}>10^{-5} \mathrm{GeV}$ and a benchmark mass with $M_{H^{0}, A^{0}}=253 \mathrm{GeV}$. We vary the mass difference between doubly charged Higgs and charge neutral Higgs by at most 
$5 \mathrm{GeV}$. In this region of the triplet vev, the gauge boson decay mode of $H^{ \pm \pm}$is predominant. The associated production cross section $p p \rightarrow H^{+} H^{0}$ varies between $\sigma \sim 17$ and $20 \mathrm{fb}$. The product of different branching ratios becomes maximal in a region $v_{\Delta} \sim 10^{-4}-10^{-2} \mathrm{GeV}$. To analyze the signal events, we implement a few basic cuts, for which we get a cut efficiency $c_{\text {eff }}=0.6$. With integrated luminosity of $\mathcal{L}=3000 \mathrm{fb}^{-1}$, we find that a doubly charged Higgs of mass around $257 \mathrm{GeV}$ can lead to 600 events at the future upgrade of the LHC.

Additionally, we also consider heavier neutral and doubly charged Higgs for which tetralepton signature can be observed in a $p p$ collider with higher c.m. energy. For illustration, we consider the mass $M_{H^{0}, A^{0}}=900 \mathrm{GeV}$ and vary the masses of doubly and singly charged Higgs at most by $M_{H^{ \pm}}-M_{H^{ \pm \pm}} \sim 5 \mathrm{GeV}$. We explore the signal sensitivity for this benchmark point at the $100 \mathrm{TeV} p p$ collider. We consider both the dilepton and gauge boson decay modes of the doubly charged Higgs. For heavier mass, the branching ratio of $H^{ \pm} \rightarrow H^{ \pm \pm} W^{*}$ is large for a very large $\lambda_{4}$. We find that the production cross section $p p \rightarrow H^{+} H^{0}$ varies nominally $\sigma \sim 5 \mathrm{fb}$. We find that in a narrow region in the $\lambda_{4}-v_{\Delta}$ plane, the same-sign tetralepton events can be very large $N_{\text {evnt }} \sim \mathcal{O}\left(10^{3}\right)$.

\section{ACKNOWLEDGMENTS}

M. M. acknowledges the support of the DST-INSPIRE research Grant No. IFA14-PH-99 and the cluster facility of the Institute of Physics, Bhubaneswar, India. E. J. C. and M. M. thank the workshop "NuHoRizon" held during February at HRI, India, where the work has been initiated. This project has received funding from the European Union's Horizon 2020 research and innovation programme InvisiblesPlus RISE under the Marie Sklodowska-Curie Grant Agreement No. 690575 and No. 674896.
[1] P. F. de Salas, D. V. Forero, C. A. Ternes, M. Tortola, and J. W. F. Valle, Status of neutrino oscillations 2018: $3 \sigma$ hint for normal mass ordering and improved $C P$ sensitivity, Phys. Lett. B 782, 633 (2018).

[2] S. Weinberg, Baryon, and Lepton Nonconserving Processes, Phys. Rev. Lett. 43, 1566 (1979).

[3] F. Wilczek and A. Zee, Operator Analysis of Nucleon Decay, Phys. Rev. Lett. 43, 1571 (1979).

[4] P. Minkowski, $\mu \rightarrow e \gamma$ at a rate of one out of $10^{9}$ muon decays?, Phys. Lett. 67B, 421 (1977).

[5] R. N. Mohapatra and G. Senjanovic, Neutrino Mass and Spontaneous Parity Violation, Phys. Rev. Lett. 44, 912 (1980).

[6] T. Yanagida, Horizontal symmetry and masses of neutrinos, Conf. Proc. C 7902131, 95 (1979).

[7] M. Gell-Mann, P. Ramond, and R. Slansky, Complex spinors and unified theories, Conf. Proc. C 790927, 315 (1979).

[8] J. Schechter and J.W.F. Valle, Neutrino masses in $\mathrm{SU}(2) \times \mathrm{U}(1)$ theories, Phys. Rev. D 22, 2227 (1980).

[9] K. S. Babu, C. N. Leung, and J. T. Pantaleone, Renormalization of the neutrino mass operator, Phys. Lett. B 319, 191 (1993).

[10] S. Antusch, M. Drees, J. Kersten, M. Lindner, and M. Ratz, Neutrino mass operator renormalization in two Higgs doublet models and the MSSM, Phys. Lett. B 525, 130 (2002).

[11] M. Magg and C. Wetterich, Neutrino mass problem and gauge hierarchy, Phys. Lett. 94B, 61 (1980).

[12] T. P. Cheng and L.-F. Li, Neutrino masses, mixings and oscillations in $\mathrm{SU}(2) \times \mathrm{U}(1)$ models of electroweak interactions, Phys. Rev. D 22, 2860 (1980).
[13] G. Lazarides, Q. Shafi, and C. Wetterich, Proton lifetime and Fermion masses in an SO(10) model, Nucl. Phys. B181, 287 (1981).

[14] R. N. Mohapatra and G. Senjanovic, Neutrino masses and mixings in gauge models with spontaneous parity violation, Phys. Rev. D 23, 165 (1981).

[15] R. Foot, H. Lew, X. G. He, and G. C. Joshi, Seesaw neutrino masses induced by a triplet of leptons, Z. Phys. C 44, 441 (1989).

[16] A. Arhrib, R. Benbrik, M. Chabab, G. Moultaka, M. C. Peyranere, L. Rahili, and J. Ramadan, The Higgs potential in the type II seesaw model, Phys. Rev. D 84, 095005 (2011).

[17] P. S. B. Dev, D. K. Ghosh, N. Okada, and I. Saha, $125 \mathrm{GeV}$ Higgs boson and the type-II seesaw model, J. High Energy Phys. 03 (2013) 150.

[18] D. Das and A. Santamaria, Updated scalar sector constraints in the Higgs triplet model, Phys. Rev. D 94, 015015 (2016).

[19] E. J. Chun, H. M. Lee, and P. Sharma, Vacuum stability, perturbativity, EWPD and Higgs-to-diphoton rate in type II seesaw models, J. High Energy Phys. 11 (2012) 106.

[20] E. J. Chun, K. Y. Lee, and S. C. Park, Testing Higgs triplet model and neutrino mass patterns, Phys. Lett. B 566, 142 (2003).

[21] A. G. Akeroyd and M. Aoki, Single and pair production of doubly charged Higgs bosons at hadron colliders, Phys. Rev. D 72, 035011 (2005).

[22] P. F. Perez, T. Han, G.-y. Huang, T. Li, and K. Wang, Neutrino masses and the CERN LHC: Testing type II seesaw, Phys. Rev. D 78, 015018 (2008). 
[23] A. Melfo, M. Nemevsek, F. Nesti, G. Senjanovic, and Y. Zhang, Type II seesaw at LHC: The roadmap, Phys. Rev. D 85, 055018 (2012).

[24] F. del Aguila and J. A. Aguilar-Saavedra, Distinguishing seesaw models at LHC with multi-lepton signals, Nucl. Phys. B813, 22 (2009).

[25] S. Chakrabarti, D. Choudhury, R. M. Godbole, and B. Mukhopadhyaya, Observing doubly charged Higgs bosons in photon-photon collisions, Phys. Lett. B 434, 347 (1998).

[26] M. Aoki, S. Kanemura, and K. Yagyu, Testing the Higgs triplet model with the mass difference at the LHC, Phys. Rev. D 85, 055007 (2012).

[27] A. G. Akeroyd and H. Sugiyama, Production of doubly charged scalars from the decay of singly charged scalars in the Higgs triplet model, Phys. Rev. D 84, 035010 (2011).

[28] E. J. Chun and P. Sharma, Search for a doubly-charged boson in four lepton final states in type II seesaw, Phys. Lett. B 728, 256 (2014).

[29] F. del Guila and M. Chala, LHC bounds on lepton number violation mediated by doubly and singly-charged scalars, J. High Energy Phys. 03 (2014) 027.

[30] S. Banerjee, M. Frank, and S. K. Rai, Higgs data confronts sequential fourth generation Fermions in the Higgs triplet model, Phys. Rev. D 89, 075005 (2014).

[31] Z. Kang, J. Li, T. Li, Y. Liu, and G.-Z. Ning, Light doubly charged Higgs boson via the $W W^{*}$ channel at LHC, Eur. Phys. J. C 75, 574 (2015).

[32] Z.-L. Han, R. Ding, and Y. Liao, LHC phenomenology of type II seesaw: Nondegenerate case, Phys. Rev. D 91, 093006 (2015).

[33] Z.-L. Han, R. Ding, and Y. Liao, LHC phenomenology of the type II seesaw mechanism: Observability of neutral scalars in the nondegenerate case, Phys. Rev. D 92, 033014 (2015).

[34] K. S. Babu and S. Jana, Probing doubly charged Higgs bosons at the LHC through photon initiated processes, Phys. Rev. D 95, 055020 (2017).

[35] A. Crivellin, M. Ghezzi, L. Panizzi, G. M. Pruna, and A. Signer, Low- and high-energy phenomenology of a doubly charged scalar, Phys. Rev. D 99, 035004 (2019).

[36] D. Borah, B. Fuks, D. Goswami, and P. Poulose, Investigating the scalar sector of left-right symmetric models with leptonic probes, Phys. Rev. D 98, 035008 (2018).

[37] R. Padhan, D. Das, M. Mitra, and A. K. Nayak, Probing doubly and singly charged Higgs at $p p$ collider HE-LHC, arXiv:1909.10495.

[38] M. Aaboud et al. (ATLAS Collaboration), Search for doubly charged Higgs boson production in multi-lepton final states with the ATLAS detector using protonproton collisions at $\sqrt{s}=13 \mathrm{TeV}$, Eur. Phys. J. C 78, 199 (2018).

[39] CMS Collaboration, A search for doubly-charged Higgs boson production in three and four lepton final states at $\sqrt{s}=13 \mathrm{TeV}$, CERN Tech. Report No. CMS-PAS-HIG16-036, 2017.

[40] M. Aaboud et al. (ATLAS Collaboration), Search for doubly charged scalar bosons decaying into same-sign $W$ boson pairs with the ATLAS detector, Eur. Phys. J. C 79, 58 (2019).

[41] V. Khachatryan et al. (CMS Collaboration), Study of Vector Boson Scattering and Search for New Physics in Events with Two Same-Sign Leptons and Two Jets, Phys. Rev. Lett. 114, 051801 (2015).

[42] A. M. Sirunyan et al. (CMS Collaboration), Observation of Electroweak Production of Same-Sign $W$ Boson Pairs in the Two Jet and Two Same-Sign Lepton Final State in Proton-Proton Collisions at $\sqrt{s}=13 \mathrm{TeV}$, Phys. Rev. Lett. 120, 081801 (2018).

[43] S. Kanemura, K. Yagyu, and H. Yokoya, First constraint on the mass of doubly-charged Higgs bosons in the same-sign diboson decay scenario at the LHC, Phys. Lett. B 726, 316 (2013).

[44] S. Kanemura, M. Kikuchi, K. Yagyu, and H. Yokoya, Bounds on the mass of doubly-charged Higgs bosons in the same-sign diboson decay scenario, Phys. Rev. D 90, 115018 (2014).

[45] S. Kanemura, M. Kikuchi, H. Yokoya, and K. Yagyu, LHC Run-I constraint on the mass of doubly charged Higgs bosons in the same-sign diboson decay scenario, Prog. Theor. Exp. Phys. 2015, 051B02 (2015).

[46] P. S. B. Dev and Y. Zhang, Displaced vertex signatures of doubly charged scalars in the type-II seesaw and its left-right extensions, J. High Energy Phys. 10 (2018) 199.

[47] M. Mitra, S. Niyogi, and M. Spannowsky, Type-II seesaw and multilepton signatures at Hadron colliders, Phys. Rev. D 95, 035042 (2017).

[48] D. K. Ghosh, N. Ghosh, I. Saha, and A. Shaw, Revisiting the high-scale validity of type-II seesaw model with novel LHC signature, Phys. Rev. D 97, 115022 (2018).

[49] T. B. de Melo, F. S. Queiroz, and Y. Villamizar, Doubly charged scalar at the high-luminosity and high-energy LHC, Int. J. Mod. Phys. A 34, 1950157 (2019).

[50] J. Abdallah et al. (DELPHI Collaboration), Search for doubly charged Higgs bosons at LEP-2, Phys. Lett. B 552, 127 (2003).

[51] J.-F. Shen and Z.-X. Li, Doubly charged Higgs bosons pair production through $W W$ fusion at high-energy $e^{+} e^{-}$linear colliders, Europhys. Lett. 111, 31001 (2015).

[52] S. Blunier, G. Cottin, M. A. Daz, and B. Koch, Phenomenology of a Higgs triplet model at future $e^{+} e^{-}$colliders, Phys. Rev. D 95, 075038 (2017).

[53] J. Cao and X.-Y. Tian, Doubly and singly charged Higgs pair production at high-energy $e^{+} e^{-}$linear colliders, Int. J. Mod. Phys. A 31, 1650056 (2016).

[54] Y.-C. Guo, C.-X. Yue, and Z.-C. Liu, The signatures of doubly charged leptons in future linear colliders, J. Phys. G 44, 085004 (2017).

[55] P. Agrawal, M. Mitra, S. Niyogi, S. Shil, and M. Spannowsky, Probing the type-II seesaw mechanism through the production of Higgs bosons at a lepton collider, Phys. Rev. D 98, 015024 (2018).

[56] E. J. Chun and P. Sharma, Same-sign tetra-leptons from type II seesaw, J. High Energy Phys. 08 (2012) 162.

[57] A. Alloul, N. D. Christensen, C. Degrande, C. Duhr, and B. Fuks, FeynRules 2.0-A complete toolbox for treelevel phenomenology, Comput. Phys. Commun. 185, 2250 (2014). 
[58] J. Alwall, R. Frederix, S. Frixione, V. Hirschi, F. Maltoni, O. Mattelaer, H.-S. Shao, T. Stelzer, P. Torrielli, and M. Zaro, The automated computation of tree-level and next-to-leading order differential cross sections, and their matching to parton shower simulations, J. High Energy Phys. 07 (2014) 079.

[59] R. D. Ball, V. Bertone, S. Carrazza, L. Del Debbio, S. Forte, A. Guffanti, N. P. Hartland, and J. Rojo (NNPDF Collabo- ration), Parton distributions with QED corrections, Nucl. Phys. B877, 290 (2013).

[60] T. Sjostrand, S. Mrenna, and P. Z. Skands, A brief introduction to PYTHIA 8.1, Comput. Phys. Commun. 178, 852 (2008).

[61] M. Dobbs and J. B. Hansen, The HepMC C++ Monte Carlo event record for high energy physics, Comput. Phys. Commun. 134, 41 (2001). 